\title{
LA PROYECCIÓN en el sector salud en Colombia
}

\section{RESUMEN}

Este artículo hace un análisis global de la situación latinoamericana con respecto al potencial de los servicios de salud y aseguramiento. Se enfoca en un segundo momento la situación particular colombiana, para luego centrarse en los beneficios, desventajas, oportunidades y diversos aspectos ya sean positivos o negativos que trae consigo la negociación del TLC en Colombia.

\section{alabras clave}

Mercado imperfecto, fondo de seguridad y garantía (Fosyga), sistema de selección de beneficiarios (Sisben), desempleo, elusión, evasión, integración vertical, Tratado de libre comercio.
Por:

Hector William Catillo Andrés M. Castro

Nelson Díaz C.

Claudia Góméz,

Sonia Gómez

$\mathbf{L}$ a situación colombiana con respecto al comercio de los servicios de salud ha mejorado sustancialmente debido a los cambios, los acuerdos y los tratados que se han llevado a cabo en el país. Uno de los temas más importantes en el que Colombia se ha enfocado es en el potencial exportador que tiene el país.

Después de haber analizado tanto los puntos fuertes como las debilidades, se han reconocido algunos productos y servicios que se pueden ofrecer al exterior.

En este sentido es evidente que el sector salud colombiano se beneficiará con el TLC. Sin embargo, es necesario profundizar en muchos de los aspectos relacionados con la IED (inversión extranjera directa), el mejoramiento de los procesos y servicios, el establecimiento de precios, la ventaja

\footnotetext{
1 Por Héctor Wilman Castillo, Andrés M. Castro, Nelson Díaz C., Claudia Gómez y Sonia Gómez, candidatos a la Maestría en Gestión de las Organizaciones EAN-UQAC; con el apoyo del profesor Mauricio Nieto Potes.
} 
competitiva, el área de cobertura, etc., con el fin de darle a Colombia un estímulo real en el área de salud y bienestar de sus habitantes.

En el tema de aseguramiento la visión es menos clara y reina mucha incertidumbre, ya que las variables de empleo con cobertura en salud y consumo gravado (IVA) tienen un comportamiento con tendencia a la baja, que con cambios del mercado productivo importantes como lo son un TLC, presentan un escenario con muchas dudas, mayor estancamiento del régimen contributivo, mayor gasto estatal para sostener el aumento de la cobertura a expensas del régimen subsidiado.

Este artículo hace un análisis global de la situación latinoamericana con respecto a estos temas, se enfoca luego en la situación particular colombiana, para luego centrarse en los beneficios, desventajas, oportuni-dades y diversos aspectos ya sean positivos o negativos que trae consigo la negociación del TLC en Colombia.

En las últimas décadas se han presen-tado muchos fenómenos y cambios en diversos campos del sector salud y del comercio de sus servicios, todos con un efecto diferente en los países latinoamericanos. Temas como la privatización, los cambios en las diversas entidades de salud y la introducción del Seguro Social, que busca un beneficio trilateral (proveedores, consumidores y aseguradores), enmarcan la problemática social colombiana de los últimos años con respecto a los servicios de salud. Aunque la mayoría de estos cambios se han realizado con el fin de mejorar, y aunque los índices de afiliación han crecido cubriendo así a una mayor parte de la población, no se han logrado los resultados esperados y, la mala calidad y la demora en los servicios siguen siendo preocupantes.

\section{A}

The Colombian situation regarding the trade of the health services has improved substantially due to all the changes, treaties and agreements that the country has undergone. One of the most important topics Colombia has focused on is its potential to export the health services and also, on what the country can offer abroad; all this, after having analyzed its weaknesses and strengths. It is obvious that the Colombian health sector will benefit from the FTA. However, it is necessary to study in depth several of the aspects dealing with the External Direct Investment, the improvement of processes and services, pricing, competitive advantages, coverage area, etc., so that a real boost in the welfare and health of Colombian people is given.

About the security issue, the vision is less clear and full of uncertainty, because the employment aspects with health protection and the tax of consumption (IVA) have a downbehaviour, which present, with important productive market changes, as the TLC, a landscape full of doubts, more stagnancy of the Contributory Politics and a larger state spending to hold the rise of the covering with the costs of the subsidiary politic.

\section{KEY WORDS}

Imperfect, market, unemployment, elusion, evasionm vercial, integration, free trade, agreement. 
Entes internacionales y locales como el Consejo Nacional de Seguridad Social en Salud (CNSSS), El Fondo de Seguridad y Granitas (FOSYGA) y el Sistema de Selección de Beneficiarios (SISBEN) han aportado mucho a la salud colombiana y se han encargado de dirigir, controlar, regular y «asegurar» la calidad de los servicios en Colombia. Además, algunos convenios como el Acuerdo General sobre el Comercio de los Servicios (AGCS) se han negociado con la OMC con el fin de expandir y abrir las puertas a nuevas oportunidades y beneficios para la población colombiana en general.

\section{FRENTE A AMÉRICA LATINA}

\subsection{El papel del Banco Mundial}

Para superar la crisis económica que comenzó alrededor de 1980, el Fondo Monetario Internacional (FMI) exigió a los gobiernos recortar los gastos en los sectores sociales, entre ellos el sector salud (los llamados ajustes estructurales). La reducción de los presupuestos de salud provocó un deterioro aún mayor en la calidad de los servicios y una disminución notable en el grado de satisfacción de proveedores y usuarios.

El Banco Mundial, para fortalecer su programa de préstamos al sector de la salud, a finales de la década de 1990 se había convertido en la agencia internacional que más fondos prestaba a los países en vías de desarrollo para ese sector. En 1999, según los precios de 1996, el Banco Mundial había concedido préstamos por un valor total de US $\$ 16.800$ millones.

Apoyado en el poder de sus recursos, el Banco Mundial ha promovido una reforma de salud basada en los principios básicos de que el sector privado es más eficiente que el sector público y de que la función del Gobierno es regular y no proveer servicios.
Siguiendo su ideología neoliberal, el Banco Mundial propone la privatización de los servicios, su descentralización, la separación de las funciones de financiación y provisión, y la universalización del acceso a un paquete de servicios mínimos que cada país debe definir de acuerdo con sus recursos y estudios de eficiencia en función de los costos.

\subsection{La privatización en América Latina}

La mayoría de los países de América Latina se han opuesto a la privatización de la prestación de servicios de salud financiados por el sector público o por sistemas de seguridad social. Brasil tiene una larga historia de contratación de servicios hospitalarios al sector privado sin fines de lucro. Otros países, como Costa Rica, El Salvador y más recientemente México, han experimentado con planes piloto de contratación pública de servicios privados. 
Chile, que había tenido un sistema nacional de salud con cobertura universal y de acceso gratuito entre 1952 y 1981, fue el primer país latinoamericano en aplicar una reforma neoliberal a principios de la década de 1980. La dictadura fragmentó el sistema nacional de salud y creó aseguradoras privadas denominadas Institutos de Salud Provisional (ISAPRE). También descentralizó la atención primaria y hospitalaria mediante su transferencia a las municipalidades y a las áreas de salud, respectivamente, y en 1986 estableció cuotas de recuperación en el sector público. Según evaluaciones realizadas, la reforma aplicada en Chile:

- Segmentó el sistema, lo que provocó una mayor inequidad en el acceso a los servicios, ya que solo los ricos tienen los recursos económicos para beneficiarse de los ISAPRE. La segmentación no mejoró la eficiencia del sistema; el gasto per cápita de los beneficiarios de los ISAPRE es aproximadamente el doble del gasto per cápita en el sector público $(22 \%$ de la población consume $46 \%$ del gasto para la salud), a pesar de que los beneficiarios de los ISAPRE son, en promedio, más jóvenes y sanos.

- Benefició a los ISAPRE, que llegaron a funcionar con márgenes de beneficio de $20 \%$ y gastos administrativos de otro $20 \%$, debido a prácticas que la superintendencia de ISAPRE no ha podido controlar, como la selección cuidadosa de sus afiliados (jóvenes y sanos) y los subsidios directos del Estado (alrededor de 4\% del gasto de los ISAPRE).

- Aumentó la inequidad entre las áreas rurales y urbanas.

- Redujo el presupuesto público del sector de la salud, que además tiene la difícil tarea de acoger a las personas que, en períodos de crisis económica, dejan de contribuir a ISAPRE y pasan a depender del sector público.

La reforma de salud de Colombia, aplicada en 1993, es la que siguió más de cerca las recomendaciones del Banco Mundial, a la vez que intentó corregir algunos de los problemas identificados durante el proceso de la reforma chilena. 


\section{EL SEGURO DE SALUD}

La introducción del seguro se realiza a través de unos agentes creados especialmente para administrarlo: las compañías aseguradoras, quienes tienen el rol de intermediarios entre los consumidores y los productores. En sentido teórico, la figura del seguro beneficia a los tres agentes participantes: consumidores, productores y aseguradores. La introducción de este mecanismo, busca esencialmente corregir el problema de la incertidumbre financiera, que enfrentan el consumidor y el productor.

Desde el punto de vista del consumidor, los usuarios del seguro reducen el riesgo de experimentar grandes pérdidas financieras asociadas a la ocurrencia de una enfermedad. Esta solución, permite que la demanda por atención médica pueda realizarse sin restricción de ingresos, ya que se desplaza el gasto en salud del individuo a la compañía de seguros, mediante el pago anticipado de cierta suma de dinero (prima), que constituye una parte regular de su gasto, sin variaciones fuertes en el corto plazo, ni exposición al deterioro en su patrimonio.

Desde el punto de vista del productor, los proveedores de servicios reciben estímulos con relación a la demanda, ya que el seguro garantiza la existencia de recursos financieros que cubran el precio de las atenciones médicas que antes corrían el riesgo de no ser pagadas.
En cuanto a los aseguradores, sus beneficios resultan del margen entre las primas que pagan los afiliados y el costo de cubrimiento del seguro. Como cualquier agente maximizador de ganancias, el asegurador está interesado en reducir sus costos; sin embargo, la naturaleza incierta ${ }^{1}$ de éstos, hace necesario el cálculo de sus riesgos ${ }^{2}$. Alrededor de este hecho, se ha desarrollado toda una teoría de seguros, cuyo sustento teórico tiene como uno de sus pilares, la denominada «ley de los grandes números»; según la cual la distribución de la tasa promedio de enfermedad en un grupo, en la medida en que este sea más grande, podrá igualarse con la probabilidad de enfermedad de toda la población ${ }^{3}$.

Con esto, los aseguradores pueden obtener estimaciones estadísticamente significativas de sus gastos ${ }^{4}$, que les permiten un mayor grado de certeza sobre sus flujos de caja y posibilitan su sostenibilidad financiera a largo plazo. Basándose en esta información, los aseguradores diseñan paquetes de cubrimientos (planes de salud ó beneficios), y calculan el monto de las primas que cobrarán a sus afiliados según el plan que escojan; diluyendo o dispersando el riesgo entre éstos.

Con el seguro de salud, también se busca corregir el fenómeno de información asimétrica que existe entre productores y

1 Que se deriva del carácter incierto y variable de la enfermedad

2 «Oferta del seguro público de salud en Colombia». Observatorio de la seguridad social, grupo de Economía de la Salud -GES-, Universidad de Antioquia, Facultad de Ciencias Económicas (Centro de investigaciones Económicas CIE-). Medellín, Septiembre de 2001, observación \#2. Pág. 2

3 «Financiamiento del seguro de salud en Colombia». Observatorio de la seguridad social. Medellín, Junio de 2002, observación \#4. Pág. 2.

4 «Derivadas del cálculo de las probabilidades de ocurrencia de enfermedad entre sus afiliados (dado que no es posible conocer el estado real de salud de los individuos), de su experiencia en manejo de riesgo y del control de costos asociados a la atención.» (Financiamiento del seguro de salud en Colombia, Observación \#4). 
comparadores; al actuar éste como un agente mejor informado que sirve para mejorar los flujos de información entre unos y otros; con lo cual se logra disminuir ó atenuar el gran poder de mercado concentrado en el vendedor. Las mejoras en la calidad de la información, implican que el gobierno ó en general, el regulador, tenga una mayor capacidad ó grado de control sobre el comportamiento del mercado y de los agentes involucrados (calidad de los servicios, equidad). Además de la medición ó seguimiento de las variables importantes del sistema de salud, la información le otorga una mayor capacidad (poder) de maniobra para corregir las fallas detectadas (externalidades).

Es importante mencionar, que no existe solo una forma de obtener el seguro y que sus características difieren entre países, dependiendo de las estructuras organizacionales dentro de las cuales se desarrollan las instituciones creadas para llevar a cabo las funciones del sistema de salud. Con este fin, se han planteado dos modelos organizacionales, que se diferencian por el grado de participación del gobierno, y por la forma de financiamiento del seguro (Jonson y Musgrove).

En el modelo denominado aseguramiento público ${ }^{1}$, el asegurador recauda ingresos con impuestos o contribuciones obligatorias a la seguridad social, y contrata los servicios médicos con proveedores públicos o privados, o los presta directamente; en este modelo, el asegurador ejerce el monopolio en el financiamiento y los afiliados no pueden elegir libremente su asegurador. En el otro modelo, el seguro privado de salud, los usuarios pagan una prima voluntaria a un asegurador que eligen libremente en el mercado, para cubrirse de un grupo específico de riesgos. El gobierno actúa en los dos modelos como ente regulador, pero sus funciones en cada uno están diferenciadas: en el primero debe obtener los fondos necesarios para la contratación de los servicios, y en el segundo, regula la competencia entre las aseguradoras y distribuye los subsidios públicos².

En términos generales, puede apreciarse que con la introducción del seguro en el mercado de servicios de salud, se establece un estrecho vínculo entre éste y el sector financiero. Independientemente de si su naturaleza es pública o privada, el seguro de salud es en sí mismo un producto financiero, intermedio dentro de la cadena de acciones encaminadas a la producción final de un servicio de salud. La complejidad de esta relación tiene serias implicaciones para el sector salud, ya que el regulador no solo deberá corregir las fallas de su propio mercado, también deberá protegerlo de los problemas que presenta el seguro. Bajo estas condiciones, los prestadores de servicios de salud, clínicas, hospitales y centros médicos, no establecen una negociación directa con el paciente, sino que compiten entre sí, dependiendo de sus capacidades específicas, para las aseguradoras las contraten.

\footnotetext{
1 Las palabras público y privadohacen referencia a la fuente de los recursos financieros y no a la naturaleza de propiedad de las entidades encargadas del aseguramiento. (Observatorio de la seguridad social, Universidad de Antioquia, Centro de Investigaciones Económicas -CIE-, observación \#4, 2002)

2 El modelo adoptado en Colombia se encuentra en punto medio entre el aseguramiento público y el seguro privado de salud. Aunque las contribuciones a la seguridad social son obligatorias, el gobierno no actúa como asegurador; las aseguradoras, al igual que los prestadores de servicios contratados por éstas, pueden ser de naturaleza pública o privada
} 


\subsection{Eficiencia}

A diferencia de lo ocurrido en Chile, el gasto para la salud en Colombia ha aumentado notoriamente: el aporte del Gobierno pasó del $1,39 \%$ del producto interno bruto en 1988 a $4 \%$ en el año 2000; además, la cuota patronal pasó de $8 \%$ a $12 \%$ del salario, con lo que también aumentó sustancialmente el gasto privado. Se calcula que entre 1984 y 1997, el gasto para la salud aumentó en $178 \%$ y el gasto por persona pasó de US $\$ 50,00$ a finales de la década de 1980 a US $\$ 90,00$ a finales de la de 1990. Este aumento tan notable del gasto no ha ido acompañado de un mayor acceso a los servicios ni de una mejor calidad, lo que indica que no ha mejorado la eficiencia.

Hay que tener en cuenta que muchas de las personas pobres que ahora están cubiertas por un seguro, recibían atención médica en los servicios públicos de salud antes de la reforma. Y que, como ya se ha explicado, muchos pobres asegurados no pueden acceder a los servicios por falta de recursos para pagar la parte que les corresponde de los pagos compartidos, entre otras causas. Además, la adjudicación de los subsidios no se ha hecho adecuadamente.

\subsection{Situación de mercados}

Uno de los principales problemas de la industria de la salud es la información asimétrica que se da entre el proveedor y el consumidor del servicio. El consumidor desconoce la complejidad de su enfermedad y los tratamientos disponibles, y no dispone del tiempo necesario para analizar la información antes de tomar sus decisiones de consumo (Cutler, 2002), por lo que debe confiar en la veracidad del informe médico ${ }^{1}$, que puede ser dictado estratégicamente ${ }^{2}$. La información asimétrica, le otorga al médico poder de mercado y de negociación, en tanto éste tiene libertad en la fijación del precio, mientras que el individuo tiene poca capacidad y tiempo para elegir. La ineficiencia de este resultado se agrava teniendo en cuenta la incertidumbre financiera a la que están sujetos los individuos ante la imposibilidad de determinar cuánto será su gasto en salud ${ }^{3}$.

En general, la intervención del gobierno se justifica con el fin de acercar los mercados a la competencia ${ }^{4}$, basándose en la acepción teórica de que ésta maximiza el bienestar de la sociedad. Sin embargo, el mercado de servicios de salud presenta particularidades como la dificultad para determinar la calidad de los servicios y la combinación de un producto heterogéneo con preferencias heterogéneas, que hacen que, aún en mercados competitivos, se llegue a resultados ineficientes. Por esta razón, la pregunta sobre bienestar social debe ser decidida en un contexto de segundo mejor.

La incapacidad de medir con certeza la calidad de los servicios aún después de ser provistos, se explica porque los resultados de los

\footnotetext{
1 Los médicos si tienen certeza sobre el problema y tratamientos que se requieren para solucionarlo.

2 Las funciones objetivo de los médicos pueden no tener en cuenta el bienestar del consumidor, teniendo incentivos para distorsionar el verdadero diagnóstico y obtener mayores ganancias.

3 Que obedece al carácter incierto de la enfermedad, dada su ocurrencia probabilística y su variabilidad (tratamientos disponibles y eficacia de los mismos) (ARROW, 1963, citado por Restrepo, 2001)

4 Corrigiendo las fallas de mercado: (i) fallas de información; (ii) monopolios naturales; (iii) externalidades; y (iV) objetivos sociales, como distribución del ingreso o calidad de los servicios. Findlay, C. 2000. «Introduction to the regulation of services» (Productivity Commission and Australian National University)
} 
procedimientos médicos son inciertos, y dependen, tanto del procedimiento en sí mismo, como de la respuesta del paciente.

Este hecho, puede producir resultados ineficientes en mercados competitivos, ya que los proveedores no tienen motivos económicos suficientes para invertir en el mejoramiento de la calidad.

De otro lado, Satterthwaite (1979) argumenta que la combinación de un producto heterogéneo ${ }^{1}$ con preferencias heterogéneas ${ }^{2}$, les da a los vendedores el poder de mercado. Los pacientes maximizan su utilidad cuando escogen el proveedor que produce el servicio que más se acerca a sus preferencias; si tienen que escoger otro, su utilidad se reduce.

Asimismo, demuestra que la competencia por diferenciación de producto a la que llegan los productores no siempre es óptima, y concluye que en general la teoría económica no ofrece una guía de políticas convenientes en mercados con diferenciación significativa de producto.

El mercado de servicios de salud también presenta externalidades negativas, ya que los tratamientos médicos de una persona pueden incidir en los de otra. Por ejemplo, alguien que no esté enterado de que posee una enfermedad contagiosa puede infectar a otra persona; igualmente, una persona que esté tomando antibióticos y deje de hacerlo, contribuye a que la bacteria desarrolle resistencia contra este medicamento.

Estas consideraciones teóricas, justifican la intervención del gobierno como ente regulador, tanto en el mercado de los servicios de salud, como en aquellos mercados que presentan fallas que van en detrimento del bienestar de los individuos. En la práctica, la forma de intervenir difiere entre países, y en general, los gobiernos se enfrentan al dilema entre la mejor forma, o la manera óptima para proveer este servicio.

Una experiencia común en el mundo es el seguro de salud, que surge como respuesta a los problemas de información presentes en el mercado y a algunas de las particularidades mencionadas. Sin embargo, su introducción puede tener implicaciones no deseables para el sector salud, por los problemas que enfrenta el seguro para ser provisto por el mercado.

1 Todos los servicios son por definición heterogéneos, en el sentido que el mismo servicio es diferente cada vez que se presta, y a diferencia de la mayoría de bienes, si el consumidor no queda satisfecho con su elección no le es posible devolverlo.

2 Los consumidores demanda diferentes tipos de servicios, no solo dependiendo de su estado de salud, sino de otro tipo de preferencias por un tipo de médico y atención particular, lugar, etc. 


\section{EL SECTOR SALUD COLOMBIA}

Desde los 90 nuestro sistema de salud que subsidiaba la oferta de los servicios de salud, pasa a subsidiar la demanda (Ley 100), cubierto por un modelo de aseguramiento. La viabilidad económica del modelo depende de la afiliación del usuario al sistema, para lo cual están diseñadas dos formas de acceder:

a. Régimen Contributivo: cubierto por el aporte del $12 \%$ de su salario entre los trabajadores y sus patronos.

b. Régimen subsidiado: cubierto por el Estado con los recursos provenientes del Impuesto al Valor Agregado (IVA), recursos que, de acuerdo con varios indicadores tales como población y nivel de pobreza entre otros, son asignados a los municipios y distritos. Éstos deben asignar el 15\% de estos recursos para pagar el seguro de su población más vulnerable, definida a través de la encuesta del Sistema de Selección de Beneficiarios (SISBEN). Existen otras fuentes de recursos como el punto de solidaridad del salario y los aportes de cajas de compensación.

El sistema del aseguramiento en salud tiene como producto el Plan Obligatorio de Salud (POS), el cual define los servicios a que tiene derecho el beneficiario y su familia en primer grado de consanguinidad. Se accede al seguro a través del pago de la Unidad de Pago Capitado (UPC). Hay UPC diferencial entre el régimen contributivo (\$386.881,20 año) y el subsidiado (\$215.712,00 año).
Dado que no se ha logrado la cobertura universal, hay aún muchos colombianos sin aseguramiento. A ellos se les define como Vinculados y son atendidos por las Empresas Sociales del Estado (ESE) o entidades públicas a nivel local y el dinero sale de aportes del estado, del Sistema General de Participaciones para subsidios a la demanda donde aparecen los recursos de impuestos de rifas, juegos, espectáculos y licores entre otros. Es de aclarar que el Vinculado es un vacío dentro del sistema que no está contemplado en el modelo de aseguramiento que consume una gran cantidad de recursos.

El asegurador recauda el dinero que aporta el afiliado que corresponde al $12 \%$ de su salario pero para efectos contables ese dinero pertenece al FOSYGA (Fondo de Seguridad y Garantía) que es un fondo de recursos administrado por terceros que coordina el uso de los recursos del sistema. Este ente, de acuerdo a la población compensada de cada asegurador que es la suma de los afiliados mas sus beneficiarios (esposa e hijos o padres) hace cada mes un cruce de cuentas con cada asegurador de acuerdo al dinero recaudado, si su población compensada supera el dinero recaudado cubre el excedente o viceversa.

La UPC que recibe el asegurador cada mes por cada miembro de la familia del afiliado y el mismo, varía de acuerdo a la edad y el riesgo de consumir servicios: 


\section{Contributivo}

\begin{tabular}{|l|c|c|}
\hline \multicolumn{1}{|c|}{ Grupo etáreo } & Estructura de costo & Valor año \\
\hline Menores de 1 año & 2.47 & $955.596,56$ \\
\hline De 1 a 4 años & 1.28 & $495.207,94$ \\
\hline De 5 a 14 años & 0.68 & $263.079,22$ \\
\hline De 15 a 44 años (Hombres) & 0.60 & $232.128,72$ \\
\hline De 15 a 44 años (Mujeres) & 1.24 & $479.732,69$ \\
\hline De 45 a 59 años & 0.81 & $313.373,77$ \\
\hline Mayores de 60 años & 2.28 & $882.089,14$ \\
\hline
\end{tabular}

\section{Subsidiado}

\begin{tabular}{|l|c|r|}
\hline \multicolumn{1}{|c|}{ Grupo etáreo } & Estructura de costo & Valor año \\
\hline Menores de 1 año & 2.47 & $1.146 .715,88$ \\
\hline De 1 a 4 años & 1.26 & $594.249,52$ \\
\hline De 5 a 14 años & 0.68 & $315.554,46$ \\
\hline De 15 a 44 años (Hombres) & 0.60 & $278.554,46$ \\
\hline De 15 a 44 años (Mujeres) & 1.24 & $575.679,23$ \\
\hline De 45 a 59 años & 0.81 & $373.048,53$ \\
\hline Mayores de 60 años & 2.28 & $1.058 .506,96$ \\
\hline
\end{tabular}

Fuente: Acuerdo 322 del 2005, por el cual se fija el valor de la Unidad de Pago por Capitación del Plan Obligatorio de Salud de los Regímenes Contributivo y Subsidiado para el año 2006.

La UPC es diferente para cada régimen. La proyección era que fueran iguales para el 2001.

\subsection{Principios del sistema}

El Sistema de Seguridad Social en Salud tiene tres principios fundamentales:

Universal: todos tienen derecho a acceder al aseguramiento, bien sea por sus aportes (contributivo) o en caso de no poder, el Estado los subsidia (subsidiado).
Solidario: el dinero que aportan todos se utiliza en la atención de los que se enferman.

Integral: cubre todas las necesidades en servicios de salud que tienen los asegurados de manera integral entendiendo los servicios de salud como integrales. 


\subsection{Actores e interacciones}

Autoridades: el ente rector del sistema es el Consejo Nacional de Seguridad Social en Salud (CNSSS, ente colegiado de representatividad nacional), que a su vez ordena al Ministerio de la Protección Social sobre las disposiciones para que las ejecute y vigile a través de los entes territoriales y de control. El Fondo de Seguridad y Garantías (FOSYGA) es el encargado de administrar el dinero del sistema. El asegurador hace el recaudo y lo canaliza al Fosyga de acuerdo a las indicaciones del mismo. Su objetivo es velar por la viabilidad financiera del modelo. Actualmente se le integra una visión de resultados en salud, la cual aún no se ha implementado.

Aseguradores: el sistema depende del aseguramiento y para este efecto, de acuerdo con la afiliación, se cuenta con las Empresas Promotoras de Salud (EPS) para el Régimen Contributivo y las Administradoras del Régimen Subsidiado (ARS). Sus ganancias son el resultado del uso adecuado de los recursos en el mantenimiento de la salud de sus afiliados. Su rentabilidad se calcula entre el 5 y el 8\% de los aportes. Con el 92 a 95\% restante del dinero se paga la operación de los demás actores (IPS, Proveedores, Profesionales de la salud), el proceso administrativo y la operación del asegurador. Es el «dueño de los pacientes", y el ordenador del gasto, por ende es el que define: dónde, quién y con qué y, a veces, cómo se ofrece la atención a sus asegurados.

El asegurador ha generado diferentes acciones en su intención de poder controlar el gasto. La integración vertical hacia delante ha sido una de sus estrategias: tienen IPS propias, operadores logísticos para la compra de medicamentos, proveedores de alimentos, ropas, producción directa de medicamentos o su importación, entre otros. Un ejemplo del liderazgo en el tema actualmente es Saludcoop con 65 empresas de servicios que son sus propios proveedores.

Las Instituciones prestadores de servicios (IPS): las hay independientes, propias del asegurador y las Empresas Sociales del Estado (ESE), su rentabilidad depende de su capacidad de negociación, el valor agregado de sus servicios y su posicionamiento. Sus servicios se pueden duplicar y la oferta supera la demanda. Las aseguradoras, cuando un servicio es muy costoso, tienden a implementar su propia infraestructura para controlar el gasto.

Proveedores: son los encargados de abastecer de insumos al sistema para su operación, medicamentos, materiales, servicios de transporte, ropas, alimentos, etc. Su rentabilidad está definida por la competencia e importancia del producto o servicio para el servicio de salud.

En el caso de productos que son monopolio, se ejerce poder frente al acceso y al precio. Los medicamentos son el mejor ejemplo. Algunos de ellos están protegidos por patentes, lo que le permite recuperar al innovador su inversión con dividendos durante un periodo definido. Desde el momento en que un medicamento se patenta tiene 20 años de protección, de los cuales generalmente consume 12 años en su desarrollo, es decir, que tiene 8 años para recuperar y obtener beneficios.

De 10 mil medicamentos patentados solo uno sale al mercado con aprobación para la indicación, lo que representa recuperar la inversión de varios medicamentos a través del aprobado (el precio de la investigación y desarrollo). De todos los 14 mil medicamentos que actualmente hay en Colombia, menos del $3 \%$ tienen patente. 
Profesionales de la salud. Es el encargado de prestar el servicio que es la expectativa del usuario. A pesar de que hay más personas que atienden al usuario tales como el o la de facturación, información, portería, y muchos más, a quien espera ver el usuario es al profesional de salud. Los otros hacen parte de la capacidad instalada para su operación de servicio y espacialmente administrativa.

Los profesionales pueden ser de outsourcing o de planta (variando las condiciones y las políticas);son los que definen el consumo, son los que evalúan y definen el manejo; su rentabilidad y autonomía depende de su nivel de dependencia del asegurador o la IPS. En el caso de depender del asegurador, generalmente debe hacer caso a sus políticas de prestación de servicios, obedeciendo a protocolos y límites ya definidos. Generalmente entre mayor independencia del profesional mayor costo al sistema ya que apela a todos lo disponible independiente de que esté o no en el POS.

\subsection{Situación actual del sistema}

\subsubsection{Cobertura}

\begin{tabular}{|l|c|c|c|c|c|c|c|c|}
\hline $\begin{array}{l}\text { Tipo de } \\
\text { Régimen }\end{array}$ & $\mathbf{2 0 0 0}$ & $\%$ & $\mathbf{2 0 0 2}$ & $\%$ & $\mathbf{2 0 0 4}$ & $\%$ & $\mathbf{2 0 0 6}$ & $\%$ \\
\hline Contributivo/1 & $13,409,000$ & $31.7 \%$ & $13,165,000$ & $30.0 \%$ & $14,460,000$ & $31.9 \%$ & 15.434 .855 & $33,0 \%$ \\
\hline Subsidiado & $9,511,000$ & $22.5 \%$ & $11,444,000$ & $26.1 \%$ & $15,553,000$ & $34.3 \%$ & 18.082 .450 & $38,7 \%$ \\
\hline $\begin{array}{l}\text { Sub - total } \\
\text { SGSSS }\end{array}$ & $22,920,000$ & $54.2 \%$ & $24,609,000$ & $56.1 \%$ & $30,013,000$ & $66.2 \%$ & 33.517 .305 & $71.7 \%$ \\
\hline $\begin{array}{l}\text { Regímenes } \\
\text { Especiales } \\
\text { (FFMM, } \\
\text { Magisterio, } \\
\text { ECOPETROL) }\end{array}$ & $1,300,000$ & $3.1 \%$ & $1,463,000$ & $3.3 \%$ & $1,789,000$ & $3.9 \%$ & 1964.436 & $4.2 \%$ \\
\hline $\begin{array}{l}\text { Población } \\
\text { Afiliada }\end{array}$ & $24,220,000$ & $57.2 \%$ & $26,072,000$ & $59.5 \%$ & $31,802,000$ & $70.2 \%$ & 35.481741 & $75.9 \%$ \\
\hline $\begin{array}{l}\text { Población } \\
\text { Total } \\
\text { Población }\end{array}$ & $42,321,000$ & $100.0 \%$ & $43,834,000$ & $100.0 \%$ & $45,325,000$ & $100.0 \%$ & 46.772 .286 & $100.0 \%$ \\
\hline $\begin{array}{l}\text { No Afiliada /2 } \\
\text { Cobertura }\end{array}$ & $18,101,000$ & $42.8 \%$ & $17,762,000$ & $40.5 \%$ & $13,523,000$ & $29.8 \%$ & 11.290 .545 & $24.1 \%$ \\
\hline
\end{tabular}

Comparación entre la población colombiana y el número de afiliados, compensados al sistema general de seguridad social en salud 2000-2006. En amarillo valores estimados, por información verbal Minprotección Social. 
La evolución del aseguramiento durante los últimos 6 años ha sido marcada por políticas enfocadas hacia la cobertura universal.

El crecimiento del aseguramiento ha sido cercano al $50 \%$ para el periodo en referencia. El crecimiento de la cobertura ha sido a expensas del régimen subsidiado, que creció en un $72 \%$, entre tanto que la variación de la población del Contributivo para el mismo periodo fue del $15 \%$. Es importante considerar que el $24 \%$ de la población no tiene aseguramiento y que son los que se definen como vinculados.

\section{Evolución de la tasa de desempleo nacional}

(septiembre) y 7 ciudades (trimestral)

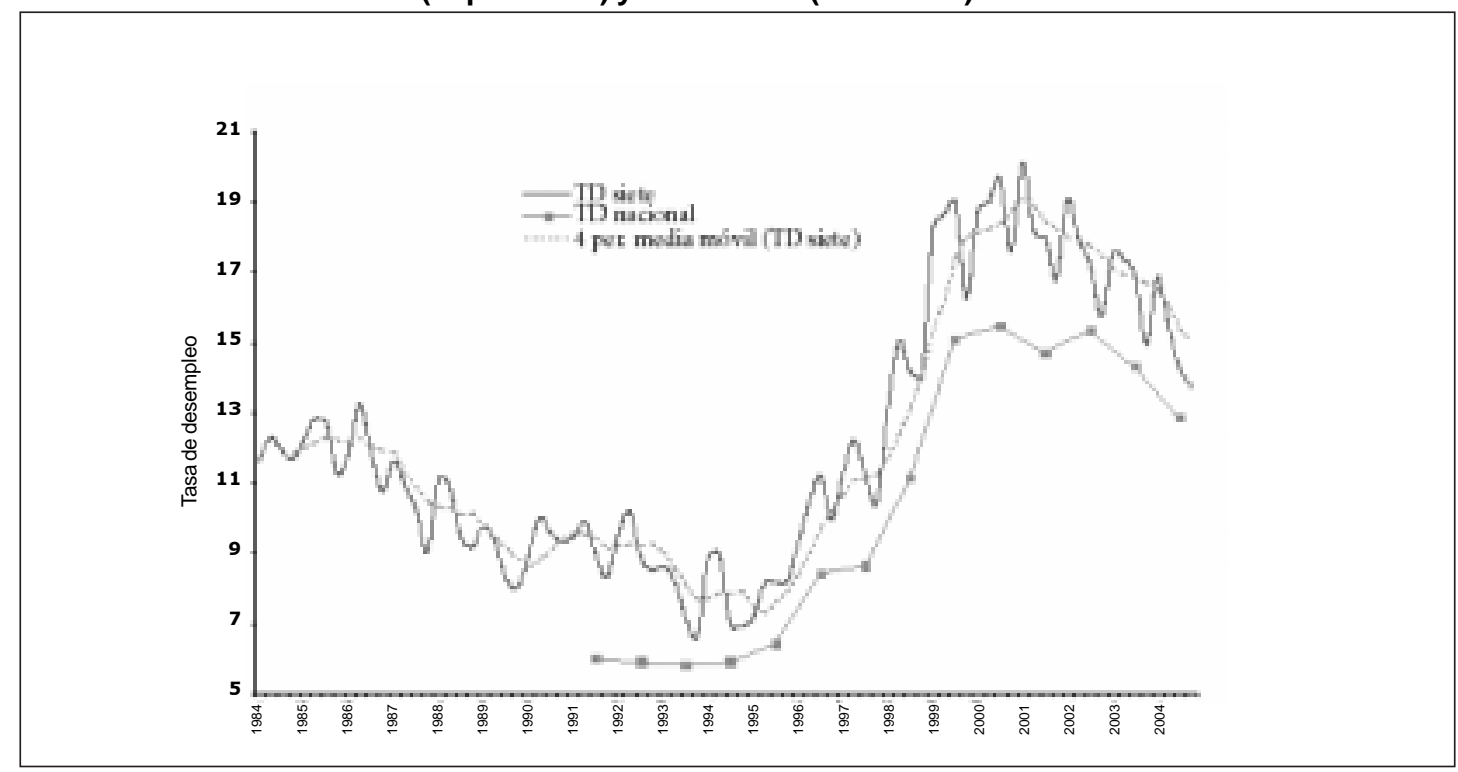

Fuente: DANE. Cálculos DNP-DDS. Para los años 1984-2000 las tasas fueron ajustadas a la ECH.

El aseguramiento del régimen contributivo tiene actualmente dos figuras, una completa y una parcial. La completa ya está definida por el POS Subsidiado, la parcial es un seguro de menor cuantía que solo cubre alto costo. Esta condición genera un espacio de selección adversa, lo que quiere decir que las personas que están fuera del sistema y tienen enfermedades de alto costo, generalmente son vinculadas al sistema y se les asigna un seguro parcial, delegando el costo y el riesgo al asegurador, los servicios de primer y segundo nivel son ofrecidos por el ente territorial (la ESE) con sus recursos de subsidio a la demanda de los vinculados.
La sostenibilidad del sistema está dada por la capacidad productiva del país, que aumente la fuerza laboral y por ende se integre más población al régimen contributivo, de tal manera que disminuya el esfuerzo fiscal del Estado. El Estado reporta una disminución significativa del desempleo para el 2006 $(11.8 \%)$ que no se ve reflejado en el aumento del aseguramiento del régimen contributivo. Lo que demuestra un crecimiento significativo del empleo informal o formal de corto plazo como la construcción, con una cultura de ahorro de los recursos evitando el aporte a salud y manteniendo la afiliación al régimen subsidiado. 


\begin{tabular}{|c|c|c|}
\hline \multicolumn{3}{|l|}{ DNP } \\
\hline POBREZA (Ingresos Día) & $\$ 5.200$ & \multirow{4}{*}{$51,8 \%$} \\
\hline IDIGENCIA (Ingresos Día) & $\$ 2.600$ & \\
\hline POBREZA (Ingresos Día) & $\$ 156.000$ & \\
\hline IDIGENCIA (Ingresos Día) & $\$ 78.000$ & \\
\hline \multicolumn{3}{|l|}{ CONTRALORIA } \\
\hline \multicolumn{2}{|l|}{ PISO DE TIERRA } & \multirow{8}{*}{$60,0 \%$} \\
\hline \multicolumn{2}{|c|}{ MATERIAL INADECUADO PAREDES } & \\
\hline \multicolumn{2}{|l|}{ SIN SERVICIOS } & \\
\hline \multicolumn{2}{|c|}{ HACINAMIENTO CRITICO (3 O MAS POR CUARTO) } & \\
\hline \multicolumn{2}{|l|}{ INASISTENCIA ESCOLAR } & \\
\hline \multicolumn{2}{|l|}{ JEFE DE HOGAR } & \\
\hline \multicolumn{2}{|c|}{ CUARTO PRIMARIA O MENOS } & \\
\hline \multicolumn{2}{|c|}{ TRES O MAS PERSONAS A CARGO } & \\
\hline
\end{tabular}

El desempleo y la pobreza son dos indicadores que nos permiten cruzar y evaluar la lógica de la cobertura del aseguramiento contributivo: si hay menos desempleo con salarios que generen capacidad de acceso a servicios de salud y educación, entonces menos pobreza, menor inversión del estado en régimen subsidiado, mayor sostenibilidad del sistema.

Porcentaje de Población en el Régimen Subsidiado sobre población en NBI por Regiones 
Paradójicamente hay menor desempleo, pero ha aumentado la pobreza, disminuido el poder adquisitivo y estancado la afiliación al contributivo. Una lectura preocupante frente al desarrollo productivo del país, el cual no ha tenido la capacidad de promover el aseguramiento contributivo sostenible.
Además si el consumo está con mayor impacto en los productos de la canasta familiar y no en los gravados con el IVA, por ende no hay ingreso de recursos por IVA y no habría cómo sostener el régimen Subsidiado. Se empieza a entender porqué la preocupación del estado en gravar a los productos de la canasta familiar a pesar del riesgo político que esto implica.

Resultados Operacionales directos EPS Acemi 1998 a 2004

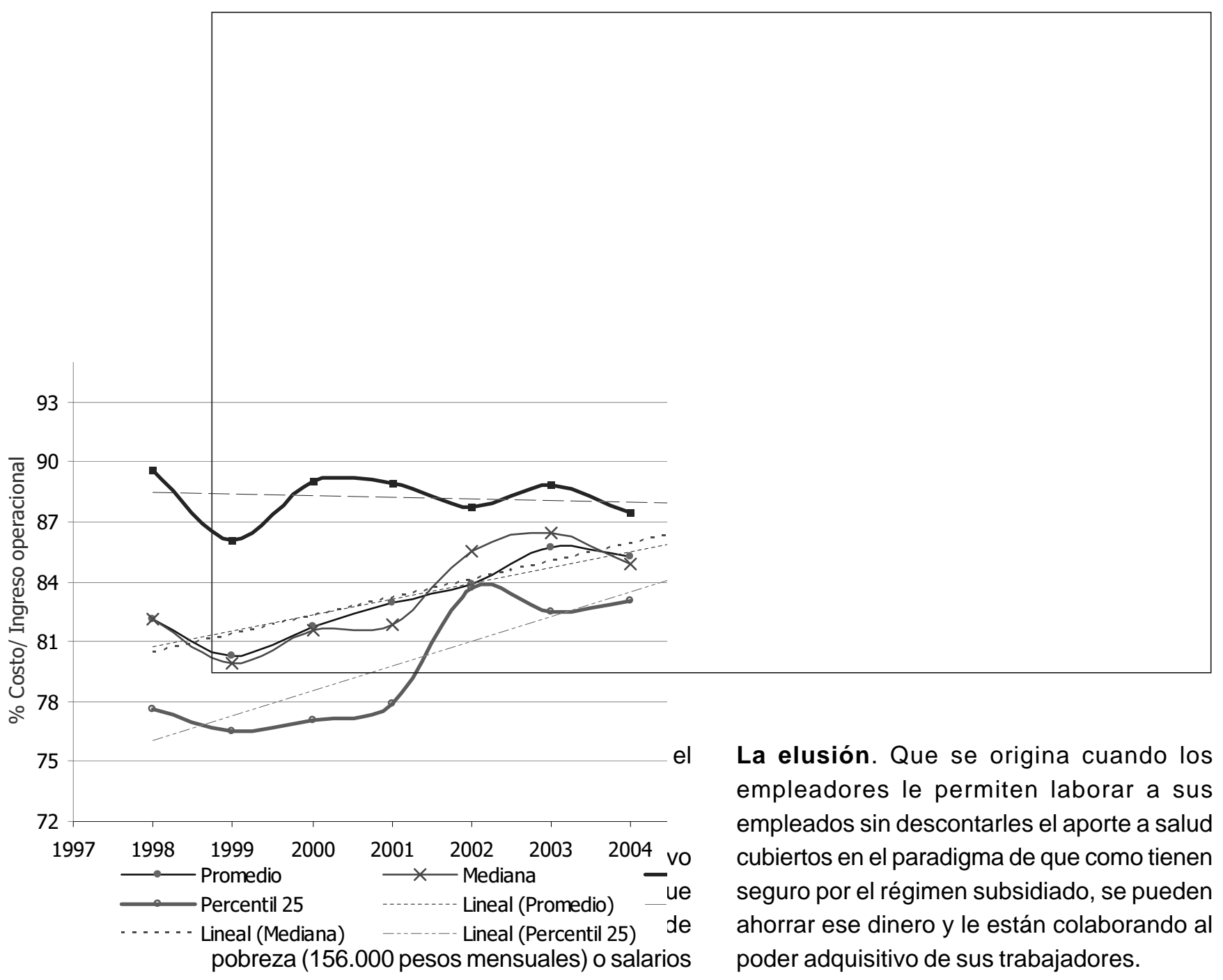




\section{Tendencia Egresos - Ingresos Operacionales}

La operación de los aseguradores tiende cada vez a ser más costosa y con un esfuerzo de control de costos que tiende a estabilizarse alrededor del $90 \%$ del ingreso. Un ejemplo es que la UPC tiene calculado un gasto en alto costo del 8\% de la UPC, que actualmente está alrededor del $18 \%$, problema que tiene mucha relación con la selección adversa que ejerce la entidad territorial (RS) o la población (en $\mathrm{RC}$ ). Se afilia primero la población enferma contrario a las prioridades de afiliación definidas en las normas. Esta dinámica hace que el equilibrio de la UPC dado por el principio de solidaridad no se dé, ya que se incrementa la población que consume servicios frente a la sana, provocando un desequilibrio financiero que afecta la viabilidad del sistema.

El efecto de una economía que aparentemente crece con un PIB de 5.2 no revela coherencia con un nivel de afiliación bajo, con un serio problema de crecimiento a expensas del subsidiado, lo que representa un fuerte esfuerzo fiscal con detrimento de la inversión social en áreas de desarrollo.
Hay un principio en el aseguramiento de la salud que nos ayuda a entender la importancia de la compensación dentro del sistema: el aumento del uso de los servicios asociado al desarrollo tecnológico, hace que el gasto tenga proyección al aumento, ya que todos los días hay tratamientos y procedimientos nuevos con mejores resultados, mas costosos; con una variable de límite definida por un presupuesto finito, la UPC, la población afiliada y la distribución del riesgo.

El asegurador es un tercero dentro del sistema que busca rentabilidad en su negocio. La salud es un bien público conexo a la vida; razón por la cual las leyes de mercado son superadas por los derechos fundamentales, por lo tanto todo colombiano dentro del sistema tiene derecho a ser atendido con la mejor opción que le ofrezca la medicina, para ello existen herramientas legales como la tutela y la acción popular.

En la mirada económica del asegurador no tiene por qué responder por servicios que no estén incluidos en su producto denominado POS, servicios que de acuerdo a la norma deben ser cubiertos en costos por el Estado (FOSYGA). La cartera por este ítem supera los 300 mil millones de pesos con tiempo entre 6 y 12 meses. 
Importancia de los Recobros en los Ingresos

de las EPS - Solo el 2004

Fuente: DPN

\begin{tabular}{|l|c|}
\hline Ingresos operacionales & 4.308 .749 .103 .594 \\
\hline Tutelas y medcamentos NO POS & 125.547 .740 .727 \\
\hline$\%$ sobre Ingresos Operacionales & $2,9 \%$ \\
\hline No pagados & 108.419 .244 .774 \\
\hline \% sobre Ingresos operacionales & $2,5 \%$ \\
\hline
\end{tabular}

Fuente: ACEMI

\begin{tabular}{|c|c|c|c|c|}
\hline Años & $\begin{array}{c}\text { Población } \\
\text { ambos } \\
\text { sexos }\end{array}$ & $\begin{array}{c}\text { Tasa global } \\
\text { de } \\
\text { Fecundidad }\end{array}$ & $\begin{array}{c}\text { Esperanza } \\
\text { de vida }\end{array}$ & $\begin{array}{c}\text { Mortalidad } \\
\text { Infantil }\end{array}$ \\
\hline 1950 & $12,568,428$ & 6,76 & 50,62 & 123,2 \\
\hline 1975 & $25,380,952$ & 4,34 & 61,72 & 73,0 \\
\hline 2000 & $42,321,386$ & 2,62 & 72,17 & 25,6 \\
\hline 2025 & $59,757,925$ & 2,15 & 76,34 & 13,8 \\
\hline 2050 & $71,549,568$ & 2,10 & 79,17 & 11,1 \\
\hline
\end{tabular}

La tendencia de los indicadores demográficos explica el porqué del crecimiento de las enfermedades crónicas y de alto costo, pero también muestran una significativa evolución del país en calidad de vida. Lo preocupante es el efecto en la productividad del país y en el aseguramiento.

\subsubsection{Debilidad de los reguladores y corrupción}

El dinero que ha ingresado al FOSYGA no se ha revertido en el fortalecimiento del sistema, sino que se ha invertido en títulos y bonos que financian otros gastos del Gobierno central. La regulación estatal en el régimen contributivo ha sido muy errática y casi inexistente. También se han observado altos niveles de corrupción en las administradoras del régimen subsidiado y en las administraciones municipales y departamentales.

Entre las manifestaciones de corrupción se encuentran los pagos de las ARS a los alcaldes para trasladar usuarios a su favor; cobros de UPC por afiliados inexistentes y carnés no entregados, pagos de prestadoras de servicios a las ARS para obtener contratos; incorporación al régimen subsidiado de personas con capacidad de pago; cobros por vacunas y otros servicios gratuitos, y retrasos en los pagos a las ARS. 


\subsubsection{Visión económica de Colombia frente al empleo y el TLC y su relación con la viabilidad del aseguramiento}

Este tema es para un debate de proceso recíproco de los efectos del TLC y el empleo. En el ámbito sociopolítico se establecen predicciones entre la izquierda y la derecha en donde las repercusiones serán mínimas: el empleo aumentará considerablemente, más la distribución del ingreso se mantendrá inalterada.

En los últimos años en América Latina, el proceso de globalización ha aumentado considerablemente. Con la llamada liberación comercial se ha orientado la oferta productiva hacia la exportación; y con mayor énfasis, en el caso colombiano ante la apertura económica de los años 90 , generando aumento de la desigualdad y el desempleo. El avance de las reformas ha generado un mayor auge comercial que financiero, trayendo consigo un aumento de demanda por trabajo no calificado, y generando brechas salariales marcadas en la mayor parte de la población económicamente activa.

Al realizar el análisis de la pobreza en Colombia luego de la reforma de los 90 y cotejarla en paralelo con el TLC actual, se establece que la liberación no insidió sobre las causas de la pobreza urbana: desempleo, informalidad y bajos ingresos.

Al realizar un comparativo del NAFTA entre EE.UU. y México, pasados 11 años de su firma, vemos reflejada la participación del PIB que en 1994 era del $38 \%$ y en el 2000 de $68 \%$, sin implicar aumento en el bienestar de las personas; ésta es una buena radiografía para el efecto colombiano en donde no solo entrarían el crecimiento en las exportaciones y la rigidez laboral, sino también problemas financieros.
Algunos cálculos del TLC y el empleo colombiano afirman que éste oscilará entre 100 mil y 300 mil empleos, índice menor que el señalado por el Estado, demostrando que hay un alto contraste entre opinión y experiencia. Se ha convertido el TLC en salud en un espejismo, pero es claro que el tratado no es la panacea para resolver los problemas colombianos en cuanto al acceso a medicamentos fiables, de calidad y precio justo. En un enfoque menos optimista se comprueba que con el índice del $11 \%$ en Colombia en el 2006, sin incluir subempleo que ha aumentado considerablemente al igual que el indicador de pobreza por encima del $50 \%$, el sostenimiento al régimen de salud presenta mucha incertidumbre.

Algunas variables claves que nos permiten ver un escenario de viabilidad del sistema:

- A nivel macro: condiciones económicas que permitan el crecimiento sostenido.

- A nivel del sistema: condiciones de la afiliación al sistema que permitan la universalización.

La sostenibilidad está determinada por:

\section{Ingresos}

- Evasión y elusión.

- Cobertura por rama ocupacional.

- Disponibilidad de recursos del presupuesto nacional.

\section{Gastos}

- Tamaño de las familias.

- Selección adversa.

- Otras prestaciones por fuera del POS.

- Leyes adicionales sin ajuste de UPC. 


\section{EXPORTACIÓN DE SERVICIOS DE SALUD}

El comercio internacional de servicios de salud se viene desarrollando desde hace muchos años, pero ha adquirido relevancia en el debate académico en la última década porque con la expansión del comercio se está negociando su liberalización en los distintos niveles de integración comercial. Actualmente, los países miembros de la Organización Mundial del Comercio (OMC) están desarrollando una nueva ronda de negociaciones en el marco del Acuerdo General sobre el Comercio de Servicios (AGCS) que es el sistema más amplio de integración de los servicios en general y, en particular, de los servicios de salud. A su vez, el desarrollo de la tecnología, las comunicaciones, las estrategias empresariales de transnacionalización, y las reformas de los sistemas de salud contribuyen a la expansión del comercio de estos servicios. Por ello, en los años recientes ha adquirido mayor relevancia el debate de las implicaciones que la liberalización y la mayor integración comercial podrían tener sobre los objetivos de salud pública.

El hecho es que, más allá de los propósitos enunciados, al llevar adelante una estrategia de liberalización del intercambio internacional de los servicios de salud, los resultados no pueden ser los deseados si la misma no va acompañada de una evaluación previa sobre los posibles impactos que se pueden verificar sobre los objetivos de equidad, calidad y eficiencia del sistema de salud. El análisis que presenta la Organización Mundial de la Salud (OMS) sobre los impactos que la liberalización del comercio de servicios de salud puede tener sobre los objetivos de equidad, calidad y eficiencia en los países en desarrollo, muestran que pueden compensarse impactos positivos en alguno de los objetivos con impactos negativos en alguno de los otros. Por lo tanto, los gobiernos deberían evaluar cada caso en particular y tomar medidas de política que busquen evitar los impactos negativos, 0 sea, los riesgos que se le generan a los sistemas de salud para alcanzar sus propósitos y aprovechar mejor los impactos positivos u oportunidades que se le presentan.

Uno de los mecanismos que se menciona como más relevante en la literatura, para evitar los impactos negativos de la liberalización, es establecer un marco regulatorio adecuado. Dado que la liberalización del intercambio de servicios implica justamente eliminar la discriminación existente en las regulaciones nacionales que afectan el ingreso y el trato nacional de los proveedores de servicios extranjeros, la OMC se ha preocupado por aclarar que liberalizar no implica desregular, sino adecuar la reglamentación existente y establecer nuevas reglamentaciones, si fuera necesario, para alcanzar los objetivos de política nacional. A su vez, no sólo es preciso contar con la regulación necesaria en cada caso particular, sino que los gobiernos cuenten con una función rectora fuerte que aplique y haga cumplir la reglamentación existente.

Varios autores sostienen que en los países en desarrollo la capacidad de regular los mercados eficazmente es muchas veces débil o limitada. Para contribuir al debate sobre los desafíos que deben enfrentar los hacedores de política de los países de América Latina y el Caribe (ALC) ante la liberalización del comercio de servicios de salud, se presentan en este trabajo tres de los desafíos que se consideran más relevantes. 
El primero, es regular adecuadamente el mercado de seguros de salud ante la liberalización del ingreso de aseguradoras extranjeras. Ello es importante para alcanzar el objetivo que generalmente persigue la liberalización del mercado de seguros de salud que es aumentar la eficiencia y evitar las posibles consecuencias negativas que pueda tener sobre la equidad en el acceso a los servicios de salud.

El segundo se refiere a la portabilidad de los seguros de salud. Un tema que presenta ciertas dificultades para su resolución pero que es relevante para que estos países desarrollen un mayor potencial exportador de servicios de salud mediante la atención a pacientes extranjeros.

El tercer desafío está relacionado con la movilidad de los profesionales. Por un lado, el problema de la «fuga de cerebros» y la necesidad de encontrar mecanismos para compensar la pérdida constante de inversión y de recursos humanos que sufren algunos países. Por otro lado, el tema de la regulación del ingreso de los profesionales que, a través del AGCS, buscan ingresar temporalmente a un país.

Para el análisis de estos desafíos, se consideró el avance que los distintos países han realizado en la liberalización de los servicios de salud a través de la firma de compromisos de liberalización en el AGCS, el proceso de integración regional de estos servicios y la participación de estos países en el comercio internacional de servicios de salud. Es importante tener en cuenta que los gobiernos pueden haber liberalizado el intercambio en un determinado servicio pero no haber consolidado un compromiso ante la OMC por las dificultades que existen para su modificación. La exportación de los servicios de salud se ha proyectado como una posibilidad atractiva para la generación de nuevos ingresos y el posicionamiento de los profesionales de la salud y los hospitales colombianos. La experiencia de nuestras IPS en mercados de seguros y la calidad de nuestros profesionales son los mayores argumentos. Por supuesto, a raíz de las negociaciones sobre el Tratado de Libre Comercio el tema toma mayor fuerza, sin embargo, con o sin TLC este será un tema de discusión a largo plazo. A partir de la implementación de la Ley 100 , el sector de la salud se comporta como un sector industrial y en ese sentido los mercados internacionales resultan atractivos, no solo porque refrescan las fuentes de recursos financieros, sino como objetivo de expansión de un sector, que a pesar de las dificultades, se muestra competitivo.

En la actualidad, existen tres tipos de posibilidades o iniciativas exportadoras en el país: la primera, corresponde a profesionales de la salud que actúan de forma independiente, atienden a pacientes extranjeros referidos, realizándoles procedimientos que, por diversas razones (económicas, confianza, disponibilidad, entre otras), decidieron no practicarse en sus países de origen. La segunda, son instituciones prestadoras, particularmente del sector privado, que mediante estrategias de alianzas o programas han buscado comercializar sus servicios en el exterior, ligados usualmente a iniciativas de promoción de ciudad. En el tercero, se encuentran las IPS que por su trayectoria y estrategia han logrado posicionar su nombre en el mercado extranjero y corrientemente exportan sus servicios, un ejemplo claro de estos es la Clínica Barraquer.

Lo anterior, indicaría que existe un potencial exportador de salud para el país, por lo menos sustentado desde la evidencia casuística, sin embargo, conviene reflexionar sobre cuál es la capacidad exportadora real y dónde están las ventajas competitivas del sector salud colombiano frente a los demás países que se encuentran en el partidor de la exportación de servicios. 


\subsection{El sector salud colom- biano frente al TLC}

A raíz de las negociaciones sobre el Tratado de Libre Comercio se ha puesto en escena el tema de la exportabilidad de los servicios de salud producidos en Colombia. Para ello revisamos primero las tendencias del sector de servicios a nivel mundial, la definición de exportación de servicios, sector salud, comercio de servicios de salud, sector salud en Colombia y finalmente un análisis de nuestro potencial exportador de estos servicios desde una visión de mercadeo.

En los últimos treinta años se ha evidenciado a nivel mundial un crecimiento en la inversión extranjera y en el comercio internacional del sector de servicios. Según el informe anual de la OMC, en el año 2004 el valor del comercio mundial de servicios comerciales aumento en $16 \%$ y alcanzó la suma de US $\$ 2.100$ mil millones de dólares.

El sector representa un lugar destacado en las economías: en el 2001 el sector representaba el $72 \%$ del PIB en los países desarrollados, el $52 \%$ del PIB en los países en desarrollo y el $57 \%$ los países de Europa Central y Oriental. La mayoría de los servicios no se pueden comercializar internacionalmente, «deben ser producidos cuando y donde se consumen», De aquí que la forma principal de llevar servicios a los mercados extranjeros sea a través de IED (inversión extranjera directa). La mayoría de los países han liberalizado los regímenes reguladores de la IED en el sector servicios.

\section{Definición de la exportación de servicios.}

Es aquella actividad económica, intangible, invisible y perecedera que exige una interacción directa entre consumidor y proveedor. Teniendo en cuenta que los servicios no se pueden comercializar internacionalmente y que por lo tanto deben ser producidos y consumidos en el lugar de origen, el tamaño del mercado en el sector servicios debe contemplar tanto el monto exportado como el ingreso de capitales que se requiere para que las empresas presten adecuadamente los servicios.

El sector social y de salud no registró participación de IED en 1990 pero en el año 2002 los países en desarrollo participaron con el $32 \%$ de la IED que recibió este sector.

\subsection{Participacion de los países en desarrollo. Entradas mundiales de IED en servicios}

En los servicios que no son comercializables internacionalmente, el crecimiento es la ventaja localizacional para la IED. Para la IED, servicios que son directamente comercializables las ventajas localizacionales se dan a través del acceso a la tecnologías de información y la comunicación, una infraestructura institucional adecuada y el poder contar con personal productivo, capacitado y a precios competitivos.

Estados Unidos ha sido durante las últimas décadas el primer importador y exportador de servicios a nivel mundial. De acuerdo con el U.S. Internacional Transactions Accounts Data, Bureau of Economic analysis, ese país importó en el año 2004 US \$263 mil millones de dólares en servicios, con un crecimiento promedio anual de $7.5 \%$ desde 1999 , similar al registrado en las importaciones de bienes

En el 2004 Colombia exportó servicios al mundo por US $\$ 1.575$ millones de dólares, con un per cápita exportador de US $\$ 35$, muy por debajo del per cápita promedio mundial (US \$333) 


\section{SERVICIOS DE SALUD}

En los últimos años, los gastos reales en atención de salud han crecido como resultado de una gran variedad de factores. A continuación se enumeran algunos de ellos:

- La difusión, cada vez mayor, de los regímenes de seguro obligatorios, a medida que se va generalizando la percepción de que la asistencia de salud, como la educación, es un derecho social básico (con excepción de los Estados Unidos, todos los países de la OCDE han logrado la cobertura universal).

- La elevación de la edad de la población en muchos países (los gastos de asistencia sanitaria de las personas de más de 65 años tienden a ser cuatro veces mayores que en los grupos de edad inferiores).

- La emergencia y el descubrimiento de nuevas enfermedades, así como la tendencia a la cronificación y a la superposición de varias patologías.

- La carencia de incentivos para que los pacientes ahorren, ya que el costo del tratamiento queda (casi) totalmente cubierto por los regímenes de seguro.

\subsection{Formas del comercio de prestación de servicios de salud}

1. Suministro transfronterizo de servicios médicos, servicios comerciales de telemedicina, servicios de telediagnóstico, envío de muestras biológicas para el diagnóstico médico a los hospitales públicos de otros países. Hasta ahora, la telemedicina se había usado sobre todo, al parecer, para sortear el obstáculo de las barreras geográficas dentro de los países y mejorar la atención de salud en regiones remotas. Ese comercio transfronterizo podría trascender los límites de la atención de salud propiamente dicha y hacerse extensivo a las funciones de gestión hospitalaria, recopilación de datos con fines estadísticos o de educación y servicios de apoyo al personal local destacado en el extranjero.

2. Consumo de servicios de salud en el extranjero. El comercio puede llevarse a cabo como movimiento de pacientes que se trasladan a) de países en desarrollo a países desarrollados, por ejemplo, lo que permite a las personas más acomodadas encontrar un acceso rápido a servicios de alta calidad en el exterior; b) de países desarrollados a países en desarrollo, con arreglo al cual los pacientes van en busca de terapias «exóticas» 0 , sencillamente, de tratamientos menos costosos cuando éstos no están cubiertos por los seguros médicos (por ejemplo cirugía estética), así como entre dos grupos de países, en aquellos casos en que, por ejemplo, los proveedores nacionales no pueden proporcionar a tiempo el servicio exigido o competir eficazmente en términos de precio o de calidad. En algunos países ofrecen precios que oscilan, con arreglo a las previsiones, entre una quinta y una décima parte de los percibidos en los países industrializados por intervenciones similares.

3. La presencia comercial, es decir, el suministro comercial de servicios de salud a través de centros o consultorios creados con ayuda de inversiones extranjeras. 


\subsection{Colombia: exportaciones de servicios de salud}

Tratado de Libre Comercio. El tema de la propiedad intelectual en las negociaciones del TLC Colombia - Estados Unidos cuenta con varios aspectos que están relacionados con la salud pública. Veamos lo que se acordó y lo que no se acordó al respecto:

Lo qué no se acordó: sobre patentes relativas a nuevos métodos de uso o nuevos usos de sustancias conocidas (impropiamente nombradas como patentes de segundos usos). Tampoco se aceptó la propuesta estado unidense de otorgar patentes sobre métodos de diagnóstico, terapéuticos y quirúrgicos para el tratamiento de personas y animales.

Lo que se acordó: compensación por retrasos irrazonables en la oficina de patentes, compensación por reducción irrazonable en el plazo efectivo de la patente en el trámite sanitario, protección de datos de prueba, vinculación del registro sanitario con la protección de patentes, cláusula para una excepción al derecho del titular de una patente y permitir a un tercero realizar ciertos actos -de ordinario prohibidos- que le aseguren entrar al mercado de manera inmediata a la expiración de la patente, el nombre común y la distintividad de la marca.

Por otra parte, es necesario tener en cuenta los efectos posibles, y es aquí donde el acuerdo internacional sobre propiedad intelectual ADPIC tiene un efecto importante sobre el costo del tratamiento para las patologías prevalentes en el país y podría generar costos adicionales para los individuos y para el país con un efecto negativo en el acceso a medicamentos exclusivos, o de monopolio.
Competencia. Es necesario tener en cuenta que Colombia no está sola en el mercado de servicios de la salud, sino que debe competir con la oferta de Estados Unidos y de los programas desarrollados por otros países latinos como Costa Rica y Cuba

Por otro lado, los representantes de las instituciones colombianas han encontrado que existen empresas extranjeras que están intentando desarrollar su oferta en Colombia no solo al buscar pacientes nacionales para llevarlos a los Estados Unidos y a otros países de Centro América, sino también prestando sus servicios a través de la presencia comercial. Es el caso de la Organización SANITAS Internacional, empresa de servicios de salud que nació en España, inició un proceso de expansión en América Latina y se instaló en Colombia como COLSANITAS S.A., la cual además de los servicios locales, ofrece servicios médicos a sus afiliados que viajan a otros países donde se encuentren establecidos (por ejemplo España y Venezuela).

Interdependencia económica. Del trabajo conjunto que deben emprender los empresarios afines con los servicios de salud, desde los puntos de atención final, pasando por los centros de investigación, los fabricantes de insumos, medicamentos, materiales quirúrgicos, equipos y muebles, hasta las universidades y las demás entidades que provean de servicios de algún tipo a las clínicas y hospitales.

La dinámica del comercio internacional en el sector de los servicios de salud demuestra su posición de extrema dependencia con otros sectores del comercio tanto de servicios como de bienes:

- Para empezar, es oportuno recordar la relación existente con los servicios 
financieros, pues muchos servicios médicos son prestados a través de pólizas de seguros de salud, por lo cual su cobertura se convierte en un elemento decisivo para la circulación internacional de pacientes.

- De igual forma, existe un vínculo comercial importante de los servicios de salud y de turismo. Desde hace varios años, en los países desarrollados se viene adelantando exitosamente esta alianza; si bien este tipo de coaliciones surge en el nivel empresarial, durante las negociaciones del ALCA y $\mathrm{OMC}$, se recomienda que los empresarios de los dos sectores desarrollen una actividad conjunta que les permita asegurar en las dos negociaciones la apertura de los modos de prestación en los mercados que les interese desarrollar.

- Finalmente, existe un nexo con el movimiento de bienes físicos en virtud de una realidad propia al funcionamiento del sector: las clínicas, los laboratorios de biotecnología y en general todas las instituciones de salud requieren, producen o reparan equipos médicos, prótesis médicas y dentales; igualmente movilizan materiales biológicos como órganos, tumores o sangre. Todas estas piezas son vitales para el funcionamiento del comercio de servicios de salud, pero están regidas por las normas internacionales del comercio de bienes.

\subsection{Tareas ya desarrolladas $y$ en proceso}

- Convenio de competitividad exportadora de servicios especializados de salud: que promueve una cultura de mejoramiento continuo y calidad en clínicas y hospitales. Fecha de la suscripción del convenio:
Medellín-Julio de 2001, durante el V Encuentro Nacional de Productividad y Competitividad.

- Decreto reglamentario 2107 de octubre 8 de 2001 referente al tema de visas.

- Decreto 2331 de octubre de 2001, que aprueba el Sistema Especial de ImportaciónExportación, para la exportación de servicios

- El Sistema Único de Acreditación en Salud avanza y se consolida como una herramienta importante para fomentar una verdadera cultura de calidad en el sector salud (se incluyó la orientación del Consejo Canadiense de Acreditación en la formulación de estándares). El sentido de la acreditación es fielmente aplicado por Icontec desde su implementación hace dos años, como resultado del concurso de méritos realizado por el Ministerio de la Protección Social.

Las normas que ajustan el Sistema Obligatorio de Garantía actual también han tomado forma para el avance del sistema, en especial las relacionadas con la definición de un sistema de información para la calidad, con un plan de ajuste a los estándares, tarea principal de la unidad sectorial de normalización (Anexo 4).

\subsection{Potencial exportador}

La exportabilidad en los servicios de salud debe abordarse como el de un producto nuevo que va a ser lanzado al mercado. Debemos establecer cuáles son las ventajas competitivas del sector frente a los demás países. Para ello desarrollamos un análisis «DOFA» el cual se presenta a continuación. 


\section{Amenazas}

- Cada país configura su propia organización de servicios de salud que se transforma en un sistema peculiar con restricciones propias. Esto impide la asimilación entre componentes de sistemas de salud diferentes (el sistema de pagos a hospitales en Colombia tiene diferencias significativas con el sistema de pagos peruano).

- El enfermo termina delegando las decisiones sobre consumo de tratamientos a terceros: médicos, aseguradores, sistemas de salud.

- Las condiciones de calidad de los servicios ofrecidos. Nuevamente los parámetros de valoración provienen del mercado externo: condiciones regulatorias y de certificación de calidad exigidas por los compradores imperantes en el mercado

- Estudios controlados como el Rand Health Experiment encontraron diferencias importantes en la elasticidad de los servicios de salud: los servicios de alta complejidad tienden a ser inelásticos, su consumo no se puede diferir e, independientemente del ingreso, cada sociedad debe darles respuesta.

- Los efectos posibles de los acuerdos internacionales sobre propiedad intelectual ADPIC sobre el costo de tratamientos generan costos adicionales con un efecto negativo en el acceso a medicamentos exclusivos o de monopolio.

- Competencia en el mercado de servicios de la salud: la oferta de Estados Unidos y otros países latinos como Costa Rica y Cuba.

\section{Oportunidades}

- La oferta es interna y se ubica en una economía mientras la demanda está en otra economía.
- La frecuencia de uso y el tamaño de la población sujeto de servicios en el mercado externo.

- El análisis del precio. El consumidor reaccionará frente al diferencial entre el precio del mercado nuevo que le es ofrecido, contra el precio de su propio mercado. Sobre esta diferencia constituye su utilidad, al adquirir en el nuevo mercado.

- Existen servicios más elásticos cuyo consumo tiende a ser discrecional y depende de las preferencias del consumidor y factores sociales. La odontología en general puede ser un buen ejemplo.

\section{Fortalezas}

- Los diferenciales de precios entre nuestro mercado de servicios y los de otros países.

- La percepción de calidad sobre nuestros profesionales de la salud, particularmente en las especialidades médicas y odontológicas.

- Experiencias exitosas de algunas instituciones locales en la exportación de servicios.

- Las clínicas colombianas empezaron a exportar sus servicios desde hace varios años. Se inició con la llegada de pacientes del exterior (especialmente de las Antillas Holandesas), quienes se sintieron atraídos por el buen nombre de algunas instituciones. Actualmente uno de los ejemplos que tenemos para mostrar es el Hospital Universitario San Vicente de Paúl.

- El Banco de Tejidos ha sido líder en el país y en Latinoamérica en trasplantes de diferentes órganos y su gestión evidencia resultados muy positivos con el soporte académico y de investigación de la Facultad de Medicina de la Universidad de Antioquia (Anexo 2). 
- El Sistema Único de Acreditación en Salud avanza y se consolida fomentando una cultura de calidad en el sector salud.

\section{Debilidades}

- La tecnología debe compararse con la existente en el mercado objetivo.

- Consumir en el nuevo mercado le representa costos adicionales. Algunos tangibles como el transporte, la hotelería, los ingresos no percibidos y otros como el lucro cesante, e intangibles tales como la valoración del tiempo laboral y familiar y la percepción del riesgo país al cual se desplaza, elemento importante en el caso de Colombia.

- La existencia de obstáculos relacionados con la circulación de personas, los requisitos en materia de nacionalidad y residencia, la concesión de licencias, el reconocimiento de la capacidad profesional, las políticas nacionales de financiación y la transferibilidad de los seguros.

\subsection{Consideraciones}

- Es necesario desarrollar un análisis diferencial por cada país y por procedimientos dentro de los servicios.
- Es necesario atraer mayor flujo de IED.

- En salud es necesario analizar las cadenas productivas desde una visión suprasectorial ya que la mayor parte de los beneficios afectarán mayormente otras industrias.

- Es necesario generar ventajas comparativas a nivel de calidad y tecnología que hagan más atractivo el servicio para la población extranjera frente a la oferta del propio país de origen o residencia permanente.

- El diferencial de precios debe ser positivo para las áreas y servicios que decidan estudiarse, pues éstas en principio deben generar incentivo económico apreciable que fomente el desplazamiento de los pacientes en Colombia.

- En caso de que el diferencial no sea sustancial o positivo, se hace necesario identificar una serie de valores agregados, como calidad, tecnología e incluso turismo que hagan más atractivo el servicio para un paciente del exterior.

- Es necesario establecer la proporción de la población de los países de interés que tendría capacidad de pago para desplazarse y pagar los servicios de salud en Colombia

- Es necesario desarrollar en el país una base de información sobre oferta y demanda externa de servicios de salud y la competencia de las instituciones y centros de investigación para el monitoreo de los mercados externos y la identificación de oportunidades. 


\section{ANEXO 1 \\ Indicadores del sector salud en 24 países de américa. Indicadores del sector salud}

\begin{tabular}{|c|c|c|c|c|c|c|c|c|c|c|}
\hline$\frac{09}{\mathbb{L}}$ & 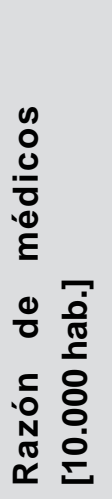 & io & 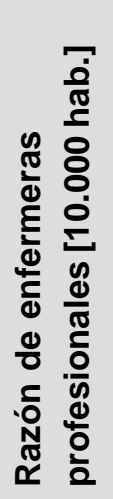 & i웅 & 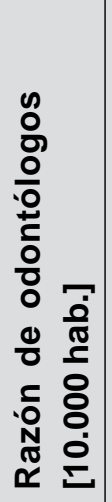 & i운 & 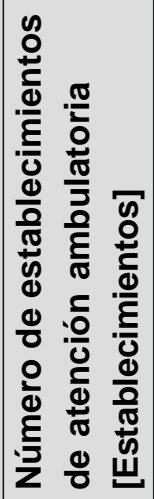 & 姿 & 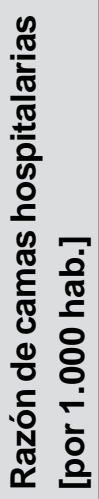 & 울 \\
\hline Argentina & 30.4 & 1998 & 5.9 & 1998 & 7.9 & 1998 & 4,534 & 2000 & 4.1 & 2000 \\
\hline Belice & 10.2 & 2000 & 12.3 & 2000 & 1.3 & 2000 & 89 & 2003 & 1.3 & 2003 \\
\hline Bermuda & 17.7 & 1999 & 89.6 & 1999 & 4.2 & 1999 & 8 & 2002 & 4.6 & 2000 \\
\hline Bolivia & 7.6 & 2001 & 3.2 & 2001 & 1.2 & 2001 & 2,588 & 2002 & 1 & 2003 \\
\hline Brasil & 20.6 & 2001 & 5.2 & 2001 & 9.5 & 2001 & 46,428 & 2002 & 2.7 & 2002 \\
\hline Canadá & 18.9 & 2002 & 73.4 & 2002 & 5.7 & 2002 & 639 & 2002 & 4.4 & 2002 \\
\hline Chile & 11.5 & 1998 & 6.6 & 2003 & 4.5 & 1999 & 1,778 & 2002 & 2.6 & 2002 \\
\hline Colombia & 12.7 & 2003 & 6.1 & 2003 & 7.8 & 2003 & 33,029 & 2001 & 1.1 & 2003 \\
\hline Costa Rica & 16 & 2000 & 3.2 & 2000 & 4.9 & 2000 & 4,503 & 2002 & 1.4 & 2003 \\
\hline Cuba & 60.4 & 2003 & 71.4 & 2003 & 8.9 & 2003 & 14,490 & 2003 & 4.9 & 2003 \\
\hline Ecuador & 16.4 & 2001 & 5.3 & 2001 & 1.7 & 2001 & 3,299 & 2002 & 1.5 & 2002 \\
\hline El Salvador & 12.6 & 2002 & 8.1 & 2002 & 5.5 & 2002 & 927 & 2003 & 0.7 & 2002 \\
\hline EE.UU. & 27.9 & 1999 & 97.2 & 1999 & 5.9 & 2000 & 4,705 & 2002 & 3.4 & 2002 \\
\hline Guatemala & 9.5 & 2003 & 3.6 & 2003 & 1.6 & 2003 & $\ldots$ & $\cdots$ & 0.5 & 2002 \\
\hline Honduras & 8.7 & 1999 & 3.2 & 1999 & 2.2 & 1999 & 2,104 & 2002 & 1 & 2002 \\
\hline México & 15.6 & 1999 & 10.8 & 1999 & 1 & 1999 & 18,506 & 2002 & 1.1 & 2002 \\
\hline Nicaragua & 16.4 & 2003 & 1.4 & 2003 & 2.9 & 2003 & 1,126 & 2003 & 0.9 & 2003 \\
\hline Panamá & 12.8 & 2001 & 10.8 & 2001 & 2.5 & 2001 & 852 & 2002 & 2.5 & 2002 \\
\hline Paraguay & 5.6 & 2002 & 2.2 & 2002 & 0.8 & 2002 & 1,279 & 2002 & 1.2 & 2002 \\
\hline Perú & 11.7 & 2000 & 8 & 2000 & 1.1 & 1999 & 7,738 & 2003 & 1.4 & 2003 \\
\hline $\begin{array}{l}\text { Puerto Rico } \\
\text { República }\end{array}$ & 17.5 & 1999 & 42.5 & 1999 & 2.5 & 1999 & 146 & 2001 & 3.3 & 2001 \\
\hline Dominicane & 19 & 1999 & 3 & 1999 & 8 & 2000 & 1,590 & 2003 & 2.1 & 2003 \\
\hline Uruguay & 39 & 2003 & 8.7 & 2003 & 12.4 & 2003 & 328 & 2002 & 1.9 & 2003 \\
\hline Venezuela & 20 & 2001 & 7.9 & 1999 & 5.7 & 2000 & 5,386 & 2003 & 0.8 & 2001 \\
\hline
\end{tabular}




\section{Continuación INDICADORES DEL SECTOR SALUD}

\begin{tabular}{|c|c|c|c|c|c|c|c|c|}
\hline$\frac{\infty}{\mathbb{K}}$ & 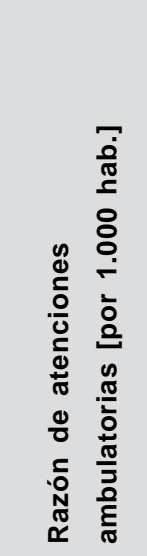 & 足 & 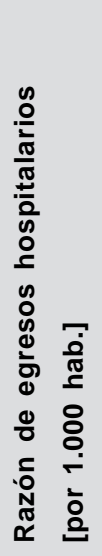 & io & 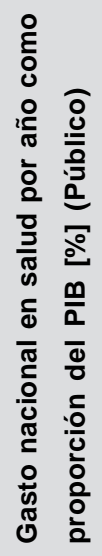 & 录 & 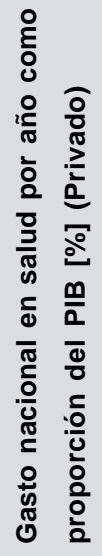 & io \\
\hline Argentina & $2,437.10$ & 2002 & 60.9 & 2002 & 4.7 & 2001 & 3.7 & 2001 \\
\hline Belice & 706 & 2003 & 82.8 & 2003 & 2.4 & 1997 & 1.4 & 1997 \\
\hline Bermuda & $1,378.20$ & 1999 & 92.4 & 2000 & $\ldots$ & & $\ldots$ & \\
\hline Bolivia & $1,687.20$ & 2002 & 43.1 & 2003 & 5.8 & 2001 & 2.9 & 2001 \\
\hline Brasil & $10,418.80$ & 2002 & 66.5 & 2002 & 3.2 & 2002 & 5.3 & 2002 \\
\hline Canadá & $6,200.00$ & 2002 & 91 & 2002 & 6.7 & 2001 & 2.6 & 2001 \\
\hline Chile & $3,900.80$ & 2003 & 101.2 & 2003 & 4.4 & 2001 & 3.9 & 2001 \\
\hline Colombia & & & 123.2 & 2000 & 5.3 & 2002 & 2.6 & 2002 \\
\hline Costa Rica & $3,199.90$ & 2003 & 79.1 & 2003 & 5.2 & 2001 & 3.4 & 2001 \\
\hline Cuba & $8,833.80$ & 2003 & 108.1 & 2003 & $\ldots$ & & & \\
\hline Ecuador & $1,600.10$ & 2002 & 55.3 & 2002 & 2.2 & 2001 & 2.4 & 2001 \\
\hline El Salvador & $1,668.00$ & 2003 & 62.3 & 2003 & 3.5 & 2001 & 4.7 & 2001 \\
\hline EE.UU. & 290.7 & 2001 & 113.4 & 2001 & 7.2 & 2000 & 5.9 & 2000 \\
\hline Guatemala & 960.1 & 2002 & 31.3 & 2002 & 1.8 & 2001 & 4.7 & 2001 \\
\hline Honduras & $1,057.40$ & 2001 & 42.8 & 2001 & 3.1 & 1998 & 2.5 & 1998 \\
\hline México & $2,592.80$ & 2002 & 61.2 & 2002 & 2.7 & 2001 & 3.3 & 2001 \\
\hline Nicaragua & $1,967.70$ & 2003 & 45.6 & 2003 & 6.8 & 2000 & 4.7 & 2000 \\
\hline Panamá & $2,281.30$ & 2002 & 76.7 & 2002 & 4.4 & 2000 & 2.2 & 2000 \\
\hline Paraguay & 558.9 & 2002 & 25.1 & 2002 & 3.5 & 2000 & 4.9 & 2000 \\
\hline Perú & $2,606.10$ & 2003 & 39.1 & 2003 & 2.8 & 2000 & 2.1 & 2000 \\
\hline $\begin{array}{l}\text { Puerto Rico } \\
\text { República }\end{array}$ & $1,430.00$ & 2001 & 109 & 2001 & $\ldots$ & $\ldots$ & $\ldots$ & $\ldots$ \\
\hline Dominicana & $1,610.70$ & 2002 & 66.1 & 2002 & 2.6 & 2000 & 3.3 & 2000 \\
\hline Uruguay & $1,774.30$ & 2003 & 45.7 & 2003 & 4.7 & 2001 & 5.6 & 2001 \\
\hline Venezuela & 714.7 & 2003 & 39.2 & 2003 & 4.1 & 2001 & 2.7 & 2001 \\
\hline \multicolumn{9}{|c|}{$\begin{array}{l}\text { FUENTE: } \\
\text { @ 2001-2004 Organización Panamericana de la Salud. Área de Análisis de Salud y Sistemas de Información } \\
\text { Sanitaria Iniciativa Regional de Datos Básicos en Salud; Sistema de Información Técnica en Salud. Washington } \\
\text { DC, 2004. Todos los derechos reservados }\end{array}$} \\
\hline \multicolumn{9}{|l|}{ Símbolos } \\
\hline \multicolumn{9}{|c|}{$\begin{array}{ll}- & \text { magnitud cero } \\
0 & \text { magnitud men } \\
\ldots & \text { dato no dispon } \\
\text { NA } & \text { No Aplicable }\end{array}$} \\
\hline
\end{tabular}

(C) 2001-2004 Organización Panamericana de la Salud. Área de Análisis de Salud y Sistemas de Información Sanitaria. Todos los derechos reservados 


\section{ANEXO 2 \\ Logros Hospital Universitario San Vicente de Paul-Medellin}

1973 - primer trasplante de riñón con donante vivo en Colombia.

1974- primer trasplante de riñón exitoso con donante cadavérico en Colombia.

1976- primer trasplante de Hígado en Latinoamérica

1976- primer trasplante de médula ósea en Colombia y Latinoamérica.

1988- primer trasplante simultáneo de riñónpáncreas en Colombia.

1993- primer trasplante de médula ósea en Colombia.

2000- primer trasplante combinado de hígadoriñón en Colombia.
2001- primer trasplante de células de cordón umbilical en Colombia.

2002- segundo trasplante de laringe en el mundo.

2003- primer trasplante de tráquea en el mundo.

2003- primer trasplante combinado de laringetráquea en el mundo.

2004- primer trasplante de intestino delgado en Colombia.

2004- primer trasplante autólogo de células progenitoras de la médula ósea por vía intracoronaria, para pacientes con enfermedad cardiaca isquémica, en Colombia.

\section{ANEXO 3 \\ Clasificación central provisional de productos CPC}

El sector de la salud comprende los servicios de la División 93.

931 Servicios de Salud Humana

9311 Servicios Hospitalarios
9312 Servicios Médicos y Dentales

9319 Otros Servicios de Salud Humana

93199 Servicios de Laboratorios Médicos

\section{ANEXO 4 Instituciones acreditadas}

A la fecha están acreditadas 8 instituciones:

- Instituto del Corazón de la Fundación Cardiovascular de Colombia, octubre 26/ 04.

- Hospital Pablo Tobón Uribe, abril 29/05.

- Hospital General de Medellín Luz Castro de Gutiérrez ESE, noviembre 11/05.
- ESE Hospital del Sur Gabriel Jaramillo Piedrahita, noviembre 30/05.

- ESE Hospital Pablo VI, febrero 14/06; Centro Policlínico del Olaya, febrero 14/ 06.

- ESE Hospital París-Acevedo-Fontidueño, febrero 14/06.

- Centro Médico Imbanaco, abril 26/06. 


\section{Bibliografia}

\begin{abstract}
BANCO INTERAMERICANO DE DESARROLLO (1998) Pluralismo estructurado: hacia un modelo innovador para la reforma de los sistemas de Salud en América Latina. Banco Interamericano de Desarrollo. Oficina del Economista Jefe. Documento de Trabajo 353. 1998.
\end{abstract}

CÁMARA DE COMERCIO DE BOGOTÁ (2005). Colombia: eExportaciones de servicios de salud. Un estudio de la Cámara de Comercio de Bogotá. Ccien nuevos productos y servicios con potencial de exportación desde Antioquia al mercado de los estados unidos. Capítulo 5: Exportación de Servicios. Estudio preparado para: ANDI Seccional Medellín. Consultores: Araujo Ibarra \& Aasociados Ss.Aa. 28 de septiembre de 2005.

CENTRO DE INVESTIGACIONES PARA EL DESARROLLO (2005). Impactos del Tratado de Libre Comercio Colombia - Estados Unidos en el sector salud del Distrito Capital. Centro de Investigaciones para el Desarrollo. Universidad Nacional de Colombia. Mayo de 2005.

CENTRO DE PROYECTOS PARA EL DESARROLLO (2005). ¿Cuál es nuestro potencial exportador en salud? Centro de Proyectos para el Desarrollo. Pontificia Universidad Javeriana. 2005. Documento de Trabajo.

CONFERENCIA DE LAS NACIONES UNIDAS SOBRE COMERCIO Y DESARROLLO. «Informe de la Reunión de Expertos en Fortalecimiento de la Capacidad y Aumento de las Exportaciones de los Países en Desarrollo en el Sector de los Servicios", Ginebra. Documento TD/B/ COM.1/7.
CUTLER, David M (2002). Health care and the public sector. National Bureau of Economic Research. Cambridge, February 2002. Pág. 26

DEPARTAMENTO NACIONAL DE PLANEACIÓN (1999). Informe de Desarrollo Humano para Colombia 1999. Departamento Nacional de Planeación. Misión Social - PNUD.

DEPARTAMENTO NACIONAL DE PLANEACIÓN (2001). Evaluación de la descentralización en salud en Colombia. Departamento Nacional de Planeación. Dirección de estudios económicos. 2001.

DEPARTAMENTO NACIONAL DE PLANEACIÓN (2004) Restricciones al comercio de servicios de salud. Departamento Nacional de Planeación. Dirección de estudios económicos. 2004.

EQUIDAD Y REFORMA EN SALUD EN COLOMBIA. Trabajo presentado en las $X$ Jornadas Colombianas de Epidemiología, Cali, Colombia, Octubre de 2000.

HOSPITAL SAN VICENTE DE PAÚL. pagina Web: ww.elhospital.org.gov

JONSON, Bengnt \& MUSGROVE, Philip. Government financing of health care in: Innovations in health care Financing. Citado en «Financiamiento del seguro de salud en Colombia. Observación \#4

LIPSON, Debra. Negociación de servicios de Salud en los tratados de comercio e integración de las Américas. Lipson, Debra. Ministerio de Salud de Canadá. Organización Panamericana de la Salud. 2002. 
MINISTERIO DE LA PROTECCIÓN SOCIAL (2005). Política Nacional de Prestación de Servicios de Salud. Ministerio de la Protección Social. Noviembre de 2005.

PRIETO, Lucia (2001). La situación del progreso social Colombiano a comienzos del Siglo XXI. Prieto, Lucia. Departamento Nacional de Planeación.

PRODUCTIVITY COMMISSION AND AUSTRALIAN NATIONAL UNIVERSITY.

Achieving better regulation of services, Canberra, 26 - 27 June. Citado por JARAMILLO, Paula. En: “¿Qué es el sector servicios, como se regula, como se comercia y cuál es su impacto en la economía?»

PUBLIC HEALTH (2005). Las reformas de salud neoliberales en América Latina: Una visión crítica a través de dos estudios de caso. Revista Panamericana de la Salud. Public Health, 2005.

RESTREPO ZEA, Jairo (2001). El seguro de salud en Colombia. ¿Cobertura Universal? Revista Gerencia y Políticas de salud. Pontificia Universidad Javeriana. 2001.

RODRÍGUEZ H, Carlos Edgar Situación actual de la acreditación en salud. Carlos Edgar Rodríguez H., MD Especialista en Administración de servicios de salud y
Gerencia Social, Magíster en Bioética, Director Nacional de Acreditación en Salud Icontec-elpulso@elhospital.org.

RUIZ GÓMEZ, Fernando; PEÑALOSA QUINTERO, Enrique; GARAVITO BELTRÁN, Liz. MModelo teórico y análisis empírico para la exportación en servicios de salud. Fernando Ruiz Gómez (1), Enrique Peñalosa Quintero (2), Liz Garavito Beltrán (3) Documento de Trabajo ASS /DT 012B- 04.

SALVADOR, Soledad (2002). La liberalización del comercio de servicios de salud en América Latina y el Caribe: principales desafíos. Salvador, Soledad. Centro Internacional de Investigaciones para el Desarrollo. Julio 2002.

SATTERTHWAITE, M. A. (1979). Consumer information, equilibrium industry price, and the number of sellers. Bell Journal of Economics. Cap. 10, Pág. 483-502. Citado en: Handbook of Health Economics. P. 1411

UNIVERSIDAD SERGIO ARBOLEDA. La salud pública y la discusión de Propiedad Intelectual en el TLC Colombia-Estados Unidos. Luis Ángel Madrid B* (c) Mayo de 2006. Universidad Sergio Arboleda. Publicado originalmente en Signo Vital edición marzo abril 2006. www.periodicoelpulso.com. Mayo de 2006. Año 7 Número 92 
